\title{
The Best Approach to Esophagectomy: Do We Know Yet?
}

\author{
Ujwal R. Yanala, $\mathrm{MD}^{1}$, Chandrakanth Are, MD MBA ${ }^{1}$, and Mashaal Dhir, $\mathrm{MD}^{2}$ \\ ${ }^{1}$ Department of Surgery, Division of Surgical Oncology, University of Nebraska Medical Center, Omaha, NE; ${ }^{2}$ Division of \\ Surgical Oncology, Department of Surgery, SUNY Upstate Medical Center, SUNY Upstate Medical University, Syracuse, \\ NY
}

Esophageal cancer is one of the top ten causes of cancerrelated mortality worldwide, with an estimated 572,034 new cases and 508,585 deaths in $2018 .^{1}$ In the US, there will be an estimated 17,650 new cases and 16,080 deaths in 2019 , making esophageal cancer the seventh leading cause of cancer-related mortality among men. ${ }^{2}$ Despite multimodal therapy, the overall 5-year survival for esophageal cancer remains low at $19 \% .^{2}$ Although surgery remains the only curative treatment, esophagectomy is associated with high perioperative morbidity $(40-50 \%)$ and mortality $(2-8 \%) .^{3}$

Pulmonary complications remain one of the major causes of morbidity and mortality after open esophagectomy (OE). Therefore, minimally invasive approaches, including laparoscopy and/or thoracoscopy, have been used to minimize the impact on pulmonary complications. Total minimally invasive esophagectomy (MIE) involves the use of both laparoscopy and thoracoscopy, whereas hybrid MIE (HMIE) most commonly involves laparoscopic mobilization combined with a thoracotomy. Nagpal et al. conducted a meta-analysis comparing the perioperative outcomes after MIE versus OE, and HMIE versus OE. ${ }^{4}$ MIE, when compared with OE, was associated with a significant reduction in blood loss, intensive care unit stay, length of stay, pulmonary complications, and total morbidity (all $p<0.05$ ). ${ }^{4}$ Similarly, HMIE demonstrated a statistically significant association with reduced blood loss, anastomotic leaks, and respiratory complications (all

\footnotetext{
(C) Society of Surgical Oncology 2019
}

First Received: 18 February 2019;

Published Online: 15 April 2019

M. Dhir, MD

e-mail:dhirm@upstate.edu $p<0.05){ }^{4}$ However, the majority of the studies were retrospective, and, as expected, there was significant heterogeneity among the studies. ${ }^{4}$

Biere et al. reported the first randomized controlled trial (RCT) of MIE versus OE from the Netherlands. ${ }^{5}$ These authors randomly assigned 56 patients to $\mathrm{OE}$ and 59 patients to MIE between 1 June 2009 and 31 March 2011, with a primary endpoint of rates of pulmonary infection within 2 weeks of surgery. ${ }^{5}$ In this small study, MIE was associated with a lower incidence of pulmonary infections at 2 weeks (9\% vs. $29 \%$ respectively; relative risk $0.3,95 \%$ CI $0.12-0.76, p=0.005$ ). However, the study has been criticized for its small sample size, higher than expected incidence of pulmonary complications in the open group, higher rates of vocal cord paralysis (14\%), and reoperations $(11 \%) .{ }^{6,7}$ Despite the limitations, this is the only RCT of MIE versus OE, and the study demonstrated a significant reduction in pulmonary complications after MIE. ${ }^{5}$

Other authors have contended that the added complexity of thoracoscopy and laparoscopy has limited the widespread adoption of MIE. ${ }^{6}$ Retrospective studies have suggested that the HMIE approach with the use of laparoscopic mobilization and open thoracotomy is associated with a reduced risk of major pulmonary complications. ${ }^{4,8}$ Another RCT by the French Eso-Gastric Tumors (FREGAT) Working Group compared HMIE with OE. ${ }^{9}$ A total of 103 patients were randomized to HMIE and 102 were randomized to $\mathrm{OE}$ from October 2009 through April 2012. ${ }^{9}$ HMIE compared with OE was associated with a significant reduction in intraoperative and postoperative complications (odds ratio [OR] 0.31, 95\% confidence interval $[\mathrm{CI}] 0.18-0.55, p<0.001) .{ }^{9}$ Additionally, the risk of pulmonary complications was 50\% lower in the HMIE group compared with OE (OR $0.50,95 \%$ CI $0.26-0.96$ ). Furthermore, there was a non-significant improvement in 3-year overall survival (OS) and 3-year disease-free 
survival (DFS) with HMIE versus OE (HMIE vs. OE, 3 -year OS $67 \%$ vs. $55 \%$, 3-year DFS $57 \%$ vs. $48 \%) .{ }^{9}$ The authors concluded that HMIE is associated with a significant reduction in intraoperative and postoperative complications, including pulmonary complications, when compared with OE, without compromising the 3-year OS and DFS. ${ }^{9}$

There have been no published RCTs of MIE or HMIE in the US. A phase II, multicenter, single-arm, prospective study by the Eastern Cooperative Oncology Group (E2202) enrolled 104 patients to assess the feasibility of MIE in a multi-institutional setting. ${ }^{10}$ These authors demonstrated that MIE is associated with a perioperative mortality of $2.1 \%$. The grade 3 or higher morbidity included anastomotic leak $(8.6 \%)$, acute respiratory distress syndrome (5.7\%), pneumonitis (3.8\%) and atrial fibrillation (2.9\%). At a median follow-up of 35.8 months, the estimated 3-year OS was $58.4 \% ;{ }^{10}$ however, population-based studies from the US are lacking.

In the current study, Naffouje et al. compared purely open transthoracic esophagectomy (OTTE, Ivor-Lewis esophagectomy) versus purely minimally invasive transthoracic esophagectomy (MITTE) using the National Surgical Quality Improvement Project (NSQIP) esophagectomy-targeted database. ${ }^{3}$ Only patients who underwent esophagectomy for cancer were included, and a propensity score design was used. ${ }^{7}$ One major strength of the study is that only patients undergoing two-field operations were included in the pure open versus minimally invasive groups. In many of the previous retrospective studies and some randomized studies, three-field and twofield operations have been combined under the open and minimally invasive groups, thus making comparisons difficult. In the propensity-matched analysis of 161 MITTE to 161 OTTE, overall complications were lower after MITTE compared with OTTE (33.5\% vs. $46 \%$; OR $0.698,95 \%$ CI $0.485-0.814, p=0.028$ ), however there was no difference in the incidence of cardiopulmonary complications, anastomotic leak, negative margins, 30-day reoperation, 30-day readmission, or 30 -day mortality. ${ }^{10}$ Although the number of lymph nodes resected was not reported, there was a higher incidence of reported abdominal and mediastinal lymphadenectomies with the OTTE. ${ }^{4}$

These findings are interesting and provocative as previous European RCTs have reported lower rates of pulmonary complications after MIE or HMIE versus OE, as well as comparable rates of lymph node retrieval. One explanation for lower reported lymphadenectomies in the NSQIP database is possible reporting bias. As there are no Current Procedural Terminology (CPT) codes for laparoscopic abdominal or mediastinal lymphadenectomies, these could have been reported as unlisted procedures by certain centers; however, this remains to be ascertained. Naffouje et al. have raised a concern about the oncologic outcomes of OTTE versus MITTE, given lower reported rates of lymphadenectomies with MITTE. Additionally, the current study is the first to report that laparoscopic MITTE is comparable with robotic MITTE with regard to perioperative outcomes.

The current study by Naffouje et al., similar to previous meta-analyses and RCTs, demonstrates the MIE is associated with a decrease in perioperative morbidity. However, the majority of the decrease in morbidity in the US population is due to a decrease in wound infection rates rather than a reduction in pulmonary complications, as seen in previous RCTs. Although administrative databases bring strength with numbers, one major limitation remains the accuracy of the data. Additionally, survival data are lacking in the NSQIP database. The current study calls for more comparative collaborative multi-institutional analysis of MIE versus OE from US centers, along with long-term oncologic outcomes. The experts need to decide whether another RCT in the US is needed when two RCTs from Europe and several other retrospective studies have shown a benefit of minimally invasive esophageal surgery for the entire operation (MIE) or part of the operation (HMIE).

DISCLOSURE The authors declare that they have no conflict of interest involving this work.

\section{REFERENCES}

1. Bray F, Ferlay J, Soerjomataram I, et al. Global cancer statistics 2018: GLOBOCAN estimates of incidence and mortality worldwide for 36 cancers in 185 countries. CA Cancer J Clin. 2018;68(6):394-424.

2. Siegel RL, Miller KD, Jemal A. Cancer statistics, 2019. $C A$ Cancer J Clin. 2019;69(1):7-34.

3. Naffouje SA Salloum RH, Khalaf Z, Salti GI. Outcomes of open vs. minimally invasive Ivor-Lewis esohpagectomy for cancer: a propensity-score matched analysis of NSQIP database. Ann Surg Oncol. Epub 29 Mar 2019. https://doi.org/10.1245/s10434-01907319-6.

4. Nagpal K, Ahmed K, Vats A, et al. Is minimally invasive surgery beneficial in the management of esophageal cancer? A metaanalysis. Surg Endosc. 2010;24(7):1621-9.

5. Biere SSAY, van Berge Henegouwen MI, Maas KW, et al. Minimally invasive versus open oesophagectomy for patients with oesophageal cancer: a multicentre, open-label, randomised controlled trial. The Lancet. 2012;379(9829):1887-92.

6. Mariette C, Robb WB. Minimally invasive versus open oesophagectomy for oesophageal cancer. Lancet. 2012;380(9845):883 (author reply 885-6).

7. Swisher S, Ajani J, Correa A, et al. Minimally invasive versus open oesophagectomy for oesophageal cancer. Lancet. 2012;380(9845):883 (author reply 885-6). 
8. Briez N, Piessen G, Torres F, et al. Effects of hybrid minimally invasive oesophagectomy on major postoperative pulmonary complications. Br J Surg. 2012;99(11):1547-53.

9. Mariette C, Markar SR, Dabakuyo-Yonli TS, et al. Hybrid minimally invasive esophagectomy for esophageal cancer. $N$ Engl $J$ Med. 2019;380(2):152-162.

10. Luketich JD, Pennathur A, Franchetti Y, et al. Minimally invasive esophagectomy: results of a prospective phase II multicenter trial-the eastern cooperative oncology group (E2202) study. Ann Surg. 2015;261(4):702-7.

Publisher's Note Springer Nature remains neutral with regard to jurisdictional claims in published maps and institutional affiliations. 